\title{
Kepler-432 b: a massive warm Jupiter in a 52-day eccentric orbit transiting a giant star $\star, \star \star, \star \star \star ~$
}

\author{
Mauricio Ortiz ${ }^{1}$, Davide Gandolfi ${ }^{1}$, Sabine Reffert ${ }^{1}$, Andreas Quirrenbach ${ }^{1}$, Hans J. Deeg ${ }^{2,3}$, Raine Karjalainen ${ }^{4}$, \\ Pilar Montañés-Rodríguez ${ }^{2,3}$, David Nespral ${ }^{2,3}$, Grzegorz Nowak ${ }^{2,3}$, Yeisson Osorio ${ }^{5}$, and Enric Palle ${ }^{2,3}$ \\ ${ }^{1}$ Landessternwarte, Zentrum für Astronomie der Universität Heidelberg, Königstuhl 12, 69117 Heidelberg, Germany \\ e-mail: mortiz@lsw.uni-heidelberg.de \\ 2 Instituto de Astrofísica de Canarias, C. Vía Láctea S/N, 38205 La Laguna, Tenerife, Spain \\ 3 Departamento de Astrofísica, Universidad de La Laguna, 38200 La Laguna, Tenerife, Spain \\ ${ }^{4}$ Isaac Newton Group of Telescopes, Apartado de Correos 321, 38700 Santa Cruz de Palma, Spain \\ 5 Nordic Optical Telescope, Apartado 474, 38700 Santa Cruz de La Palma, Spain
}

Received 11 October 2014 / Accepted 28 November 2014

\section{ABSTRACT}

\begin{abstract}
We study the Kepler object Kepler-432, an evolved star ascending the red giant branch. By deriving precise radial velocities from multi-epoch high-resolution spectra of Kepler-432 taken with the CAFE spectrograph at the $2.2 \mathrm{~m}$ telescope of Calar Alto Observatory and the FIES spectrograph at the Nordic Optical Telescope of Roque de Los Muchachos Observatory, we confirm the planetary nature of the object Kepler- $432 \mathrm{~b}$, which has a transit period of 52 days. We find a planetary mass of $M_{\mathrm{p}}=5.84 \pm 0.05 M_{\text {Jup }}$ and a high eccentricity of $e=0.478 \pm 0.004$. With a semi-major axis of $a=0.303 \pm 0.007 \mathrm{AU}$, Kepler-432 b is the first bona fide warm Jupiter detected to transit a giant star. We also find a radial velocity linear trend of $\dot{\gamma}=0.44 \pm 0.04 \mathrm{~m} \mathrm{~s}^{-1} \mathrm{~d}^{-1}$, which suggests the presence of a third object in the system. Current models of planetary evolution in the post-main-sequence phase predict that Kepler- $432 \mathrm{~b}$ will be most likely engulfed by its host star before the latter reaches the tip of the red giant branch.
\end{abstract}

Key words. planets and satellites: detection - planets and satellites: fundamental parameters - planets and satellites: gaseous planets planets and satellites: general - stars: individual: Kepler-432 (KOI-1299, KIC 10864656)

\section{Introduction}

The number of Jupiter-like planets found to orbit evolved stars has constantly been growing in recent years. This includes planets around subgiant (Johnson et al. 2010, 2011) and giant stars (Gettel et al. 2012; Sato et al. 2013; Trifonov et al. 2014). These discoveries have provided evidence that the gas-giant planet population around evolved stars possesses different orbital properties than the population orbiting main-sequence (MS) stars (e.g., Jones et al. 2014). The most notable trend is the apparent lack of close-in Jupiter-like planets orbiting giant or subgiant stars, although these objects are easily found around many MS stars by Doppler surveys (e.g., Jones et al. 2013). Specifically, there seems to be a lack of planets around giant stars with semi-major axis $a<0.5 \mathrm{AU}$. Exceptions to this apparent trend are the recently discovered planet HIP 67851 b (Jones et al. 2015) and Kepler-91 b, the only hot Jupiter known to transit a giant star (Lillo-Box et al. 2014).

There are two different mechanisms that have been proposed to explain the paucity of close-in Jupiter-like planets around giant stars. The first one states that, as a result of stellar evolution, the inner planets are tidally engulfed by their host stars as the

* Based on observations collected at the German-Spanish Astronomical Center, Calar Alto, jointly operated by the MaxPlanck-Institut für Astronomie (Heidelberg) and the Instituto de Astrofísica de Andalucía (IAA-CSIC, Granada).

$\star \star$ Based on observations obtained with the Nordic Optical Telescope, operated on the island of La Palma jointly by Denmark, Finland, Iceland, Norway, and Sweden, in the Spanish Observatorio del Roque de los Muchachos of the Instituto de Astrofísica de Canarias.

$\star \star \star$ Table 3 is available in electronic form at http://www . aanda.org outer planets move farther out (Kunitomo et al. 2011; Adamów et al. 2012; Schlaufman et al. 2013). The second one suggests that although giant planets may form around intermediate-mass stars, they do not migrate inwards, owing to the short dissipation time-scale of protoplanetary disks (Kretke et al. 2009; Currie 2009). By searching specifically for close-in Jupiter-like planets around giant stars, we can help to place constraints on the theoretical models that try to explain these observations and, possibly, also learn something about the evolution of planetary systems after the host star leaves the MS.

In this Letter, we confirm and characterize Kepler-432 b, a massive warm gas-giant planet orbiting a star ascending the red giant branch (RGB).

\section{High-resolution spectroscopic follow-up}

The radial velocity (RV) follow-up of Kepler-432 was carried out between June and October 2014 using the Calar Alto Fiber-fed Échelle spectrograph (CAFE; Aceituno et al. 2013) mounted at the $2.2 \mathrm{~m}$ telescope of Calar Alto Observatory (Almería, Spain) - and the FIbre-fed Échelle Spectrograph (FIES; Telting et al. 2014) - mounted at the $2.56 \mathrm{~m}$ Nordic Optical Telescope of Roque de los Muchachos Observatory (La Palma, Spain). We acquired $11 \mathrm{RVs}$ with CAFE $(R \sim$ $62000)$, and 16 with FIES $(R \sim 67000)$ at different epochs. To remove cosmic-ray hits, three consecutive exposures were usually taken per epoch observation. Following the observing strategy described in Aceituno et al. (2013) and Buchhave et al. (2010), we traced the RV drift of CAFE and FIES by acquiring long-exposed ( $\left.T_{\exp }=60-80 \mathrm{~s}\right)$ ThAr spectra immediately before and after each epoch observation. The data were reduced using IRAF and IDL standard routines, which include bias subtraction, 
flat fielding, order tracing and extraction, and wavelength calibration. Radial velocities were derived via multi-order crosscorrelation with the RV standard stars HD 182572 (CAFE) and HR 5777 (FIES).

The CAFE and FIES RVs are listed in Table 3 - along with their uncertainties, total exposure times, signal-to-noise (S/N) ratios per pixel at $5500 \AA$, and cross-correlation function (CCF) bisector spans - and are plotted in Fig. 1 together with the Keplerian fit to the data (upper panel) and residuals to the fit (middle panel). The lower panel of Fig. 1 shows the FIES CCF bisector spans plotted against the RV measurements, assuming that the error bars of the former are twice those of the latter. We followed the method described in Loyd \& France (2014) to account for the uncertainties of our measurements and found a $\sim 50 \%$ probability that an uncorrelated set of points (null hypothesis) can reproduce the data. The lack of a significant correlation between the CCF bisector spans and the RVs indicates that the Doppler shifts observed in Kepler-432 are most likely induced by the orbital motion of a planet and not by a blended eclipsing binary or stellar activity (see, e.g., Queloz et al. 2001). Moreover, the analysis of the centroid motion during the transit reported in the Kepler data validation report excludes, at the $3 \sigma$ level, any scenario in which the transit signal is caused by a contaminating eclipsing binary at a distance of more than $0.4^{\prime \prime}$ from the source.

\section{Results}

\subsection{Stellar properties}

We determined the spectral parameters of Kepler-432 by fitting the co-added FIES spectrum $(S / N \sim 145$ per pixel at $5500 \AA$ ) to a grid of synthetic spectra calculated with the SPECTRUM code (Gray \& Corbally 1994) using ATLAS9 models (Castelli \& Kurucz 2004). Microturbulent ( $\left.v_{\text {micro }}\right)$ and macroturbulent $\left(v_{\text {macro }}\right)$ velocities were derived following Hekker \& Meléndez (2007). Stellar mass and radius were determined via the asteroseismic scaling relations given by White et al. (2011), using our estimate for the effective temperature along with the large frequency separation and the frequency at which oscillations have the maximum power, as derived by Huber et al. (2013). We estimated the stellar age using theoretical isochrones from Bressan et al. (2012). Distance and interstellar extinction were calculated following the method described in Gandolfi et al. (2008). The derived stellar parameters are listed in Table 1.

\subsection{Orbit and planet parameters}

We fitted a Keplerian orbit to the RV data using the IDL code RVLIN (Wright \& Howard 2009). Uncertainties of the derived parameters were estimated using the bootstrap procedure described in Wang et al. (2012). Orbital period $P_{\text {orb }}$ and mid-transit epoch $T_{0}$ were fixed to the values reported in the Kepler objects of interest (KOI) database.

We fitted for the eccentricity $e$, argument of periastron $\omega$, radial velocity semi-amplitude $K$, periastron time $T_{\mathrm{p}}$, systemic RV $\gamma_{\text {sys }}$, fixed zero point RV offset between CAFE and FIES data-sets, and RV linear trend $\dot{\gamma}$. Fixing $\dot{\gamma}=0$ leads to a poor fit to the data $\left(\chi_{\text {red }}^{2}=3.5\right)$, with a systematic offset from the RV measurements. The FIES RV residuals - that is, those with the smaller error bars - show a significant correlation with time if no trend is considered, the correlation coefficient being 0.86 with a false-alarm probability lower than $0.9 \%$. Therefore, we consider the trend in the RVs to be real and obtain a value of $\dot{\gamma}=0.44 \pm 0.04 \mathrm{~m} \mathrm{~s}^{-1} \mathrm{~d}^{-1}$. This is significant at the $11 \sigma$ level and most likely due to an additional companion in the system,
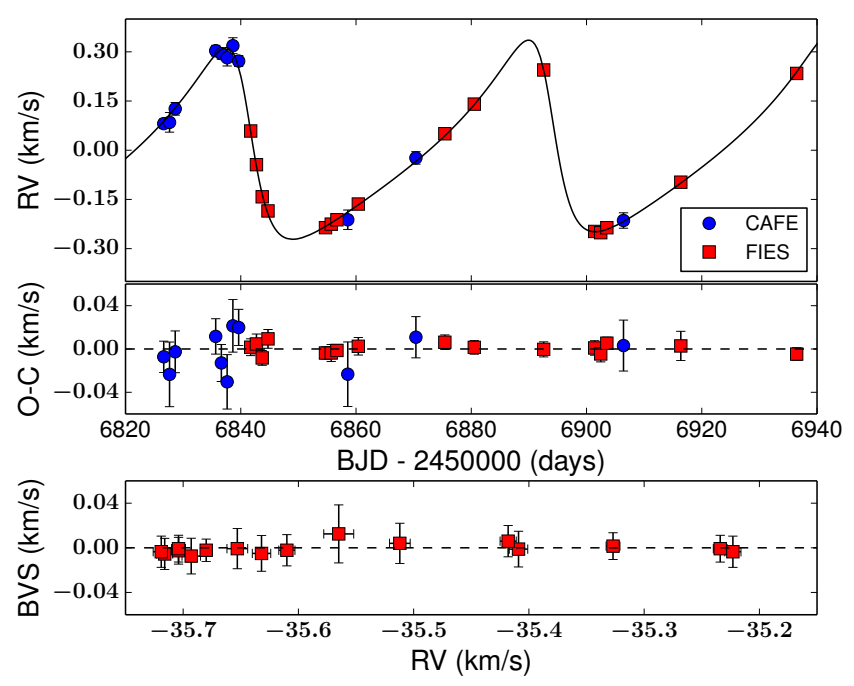

Fig. 1. Radial velocity measurements of Kepler-432. Upper panel: CAFE (blue circles) and FIES (red squares) RVs, and Keplerian fit to the data (black solid line) - including the linear RV trend. Middle panel: RV residuals. The $\mathrm{rms}$ is $\sim 17 \mathrm{~m} / \mathrm{s}$ and $\sim 5 \mathrm{~m} / \mathrm{s}$ for the CAFE and FIES data, respectively. The observed rms of the FIES RVs is consistent with the expected value of $\sim 6 \mathrm{~m} / \mathrm{s}$ for a star with $\log g=3.35 \mathrm{dex}$ (Hekker et al. 2008; Nowak 2013). Quadratically adding a jitter of $6 \mathrm{~m} / \mathrm{s}$ to our formal RV measurement errors does not change the derived orbital parameters significantly $(<1 \sigma)$. Additionally, a fit to the FIES data alone yields consistent results within $1 \sigma$. Lower panel: bisector velocity spans (BVS) of the FIES CCF versus RVs, assuming that the error bars of the former are twice those of the latter.

Table 1. Stellar parameters of Kepler-432.

\begin{tabular}{lc}
\hline \hline Parameter & Value \\
\hline Effective temperature $T_{\text {eff }}(\mathrm{K})$ & $5020 \pm 60$ \\
Spectroscopic surface gravity $\log g\left(\log _{10} \mathrm{~g} \mathrm{~cm}^{-2}\right)$ & $3.35 \pm 0.07$ \\
Metallicity [M/H] $($ dex $)$ & $-0.02 \pm 0.06$ \\
Microturbulent velocity $v_{\text {micro }}\left(\mathrm{km} \mathrm{s}^{-1}\right)$ & $1.3 \pm 0.3$ \\
Macroturbulent velocity $v_{\text {macro }}\left(\mathrm{km} \mathrm{s}^{-1}\right)$ & $3.5 \pm 0.5$ \\
Projected rotational velocity $v \sin i\left(\mathrm{~km} \mathrm{~s}^{-1}\right)$ & $\leq 1$ \\
Stellar mass $M_{\star}\left(M_{\odot}\right)$ & $1.35 \pm 0.10$ \\
Stellar radius $R_{\star}\left(R_{\odot}\right)$ & $4.15 \pm 0.12$ \\
Age $(\mathrm{Gyr})$ & $3.6_{-0.6}^{+1.0}$ \\
Distance $(\mathrm{pc})$ & $874 \pm 30$ \\
Interstellar extinction $A_{\mathrm{V}}(\mathrm{mag})$ & $0.26 \pm 0.04$ \\
Spectral type & $\mathrm{K} 2 \mathrm{III}$ \\
\hline
\end{tabular}

whose nature remains to be established. We report the best-fit orbital parameters in Table 2.

\section{Discussion}

\subsection{Internal structure and equilibrium temperature}

Kepler-432 $\mathrm{b}$ is the first bona fide confirmed transiting warm Jupiter found to orbit a red giant star. About $70 \%$ of the known transiting giant planets have densities in the range $0.35-1.20 \mathrm{~g} \mathrm{~cm}^{-3}$ and masses between 0.3 and $3 M_{\text {Jup }}$, with a peak around $1 M_{\text {Jup }}$ (Fig. 2). With $M_{\mathrm{p}}=5.84 \pm 0.05 M_{\text {Jup }}$ and $\rho_{\mathrm{p}}=5.4 \pm 0.5 \mathrm{~g} \mathrm{~cm}^{-3}$, Kepler-432 $\mathrm{b}$ is one of the most dense and massive gas-giant planets known so far. The mass of Kepler-432 b agrees with the general trend found by Doppler surveys, that is, planets around giant stars tend to be more massive $\left(3-10 M_{\text {Jup }}\right.$ ) than planets orbiting solar-like stars (see e.g., Döllinger et al. 2009; Reffert et al. 2015). In fact, around 96\% of the known planets orbiting solar-type MS stars have masses lower than $5 M_{\text {Jup }}$. 
Table 2. Orbital parameters of Kepler-432 b.

\begin{tabular}{lc}
\hline \hline Parameter & Value \\
\hline RV semi-amplitude $K(\mathrm{~m} / \mathrm{s})$ & $294.6 \pm 2.1$ \\
Eccentricity $e$ & $0.478 \pm 0.004$ \\
Argument of periastron $\omega(\mathrm{deg})$ & $68.4 \pm 0.7$ \\
Periastron time $T_{\mathrm{p}}(\mathrm{BJD}-2450000)$ & $6841.06 \pm 0.03$ \\
Systemic velocity $\gamma_{\text {sys }}\left(\mathrm{km} \mathrm{s}^{-1}\right)$ & $-33 \pm 0.3$ \\
CAFE-FIES offset velocity $(\mathrm{m} / \mathrm{s})$ & $634 \pm 5$ \\
RV linear trend $\dot{\gamma}(\mathrm{m} / \mathrm{s} / \mathrm{d})$ & $0.44 \pm 0.04$ \\
Planet mass $M_{\mathrm{p}}\left(M_{\text {Jup }}\right)$ & $5.84 \pm 0.05$ \\
Semi-major axis $a(\mathrm{AU})$ & $0.303 \pm 0.007$
\end{tabular}

Notes. Period and mid-transit epoch fixed to $P_{\text {orb }}=52.5010768$ days and $T_{0}=5004.519$ (BJD-2 450000 ). To derive the true planet mass, we use an orbital inclination value of $i=89.95^{\circ}$ from the KOI database.

We investigated the internal structure of Kepler-432 b using the models from Fortney et al. (2007), which couple planetary evolution to stellar irradiation for $\mathrm{H}-\mathrm{He}$-rich planets (dashed blue lines in Fig. 2). The planet radius of $R_{\mathrm{p}}=1.102 \pm 0.032 R_{\mathrm{Jup}}$ is consistent within $2 \sigma$ with theoretical values for giant planets with core masses of $\lesssim 100 M_{\oplus}$. This implies that the planet core accounts most likely for $6 \%$ or less of the total mass, that is, similar to the fractional core mass of Jupiter (Saumon \& Guillot 2004). We note that the solar-like metallicity of the host star $[\mathrm{Fe} / \mathrm{H}]=-0.02 \pm 0.06$ dex supports the low-fractional core mass scenario for Kepler-432 b.

As a consequence of the high eccentricity of the orbit $(e=$ $0.478 \pm 0.004)$, the planet is at nearly $\sim 0.16 \mathrm{AU}\left(\sim 8 R_{\star}\right)$ from its host star during periastron, receiving a flux of $F_{\text {per }}=(6.1 \pm$ $0.6) \times 10^{8} \mathrm{erg} \mathrm{s}^{-1} \mathrm{~cm}^{-2}$. At apastron, Kepler-432 b travels as far out as $\sim 0.45 \mathrm{AU}\left(\sim 23 R_{\star}\right)$, receiving an incoming radiation of $F_{\text {apo }}=(7.6 \pm 0.8) \times 10^{7} \mathrm{erg} \mathrm{s}^{-1} \mathrm{~cm}^{-2}$. The timeaveraged incident flux on Kepler-432 b is $\langle F\rangle=(1.66 \pm 0.17) \times$ $10^{8} \mathrm{erg} \mathrm{s}^{-1} \mathrm{~cm}^{-2}$.

Assuming a black-body stellar emission and planetary heat redistribution factor $f$ between 0.25 (instantaneous energy redistribution into the planet atmosphere) and 0.67 (instantaneous energy reradiation to space; see López-Morales \& Seager 2007), we derive an average equilibrium temperature of $T_{\mathrm{eq}}=943 \pm$ $20 \mathrm{~K}$ for a bond albedo of 0.27 (based on Kane \& Gelino 2010). Following the planet classification in Sudarsky et al. (2000), Kepler-432 b would be a member of the class IV planets with temperatures in the range $900<T_{\text {eq }}<1500 \mathrm{~K}$, for which a tropospheric silicate layer is expected to exist. However, we note that eccentric orbits can lead to significant changes in the atmospheric compositions, owing to the large variation of the incident stellar flux (Sudarsky et al. 2005). For Kepler-432 b, we expect temperature differences of $\sim 500 \mathrm{~K}$ between periastron and apastron.

\subsection{Kepler-432 b: a close-in eccentric planet}

Among planets orbiting giant stars, Kepler-432 b is very peculiar both in terms of eccentricity and orbital period, as it occupies scarcely populated regions of the $P_{\text {orb }}$ versus $M_{*}$ and $a$ versus $e$ diagrams (Fig. 3). While planets with orbital periods between 1 and $10^{4}$ days are common around MS stars, there is a clear lack of short-period planets around giant stars, and Kepler-432 b is one of the few inhabitants of the region with $P_{\text {orb }}<100$ days.

The value of $e=0.478 \pm 0.004$ for the eccentricity is among the highest for planets orbiting giant stars. Most planets around giant stars tend to have low eccentricity (63\% have $e<0.2)$, with a median of $e=0.15$, whereas planets around MS stars tend to be more eccentric. If we consider objects with $a \gtrsim 0.5 \mathrm{AU}-$ where

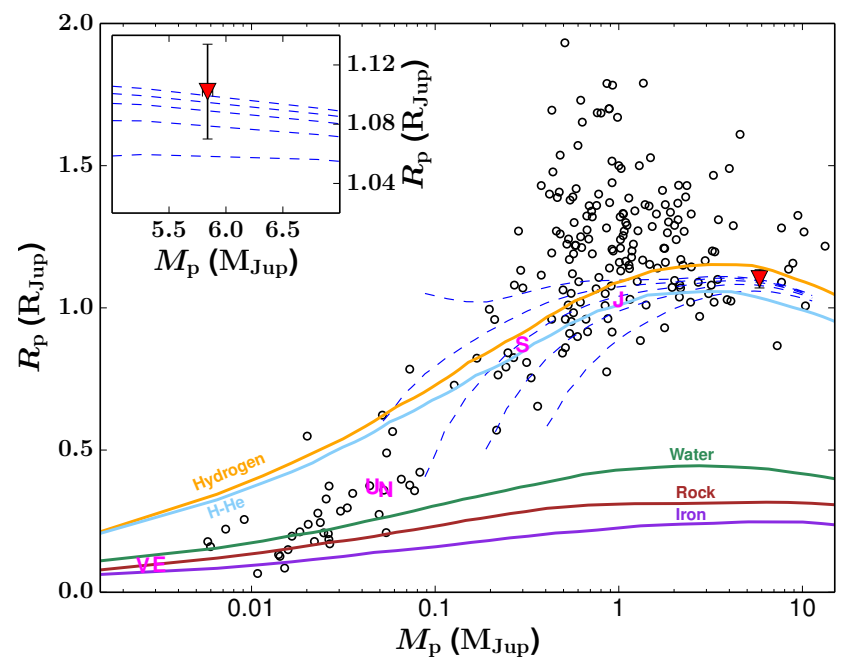

Fig. 2. Radius and masses of the known transiting exoplanets (black empty circles). The position of Kepler- $432 \mathrm{~b}$ is marked by the red triangle. The Fortney et al. (2007) isochrones for planet core masses of $0,10,25,50$, and $100 M_{\oplus}$ - interpolated to the insolation and age of Kepler-432 b - are overplotted with dashed lines from top to bottom. The upper left inset is a zoom around Kepler-432 b. Also shown are models for planets of different compositions derived by Seager et al. (2007). Solar system planets are marked with magenta letters. We note that Kepler- $432 \mathrm{~b}$ falls in a region with a lack of planets between $\sim 4.5$ and $\sim 7 M_{\text {Jup }}$.

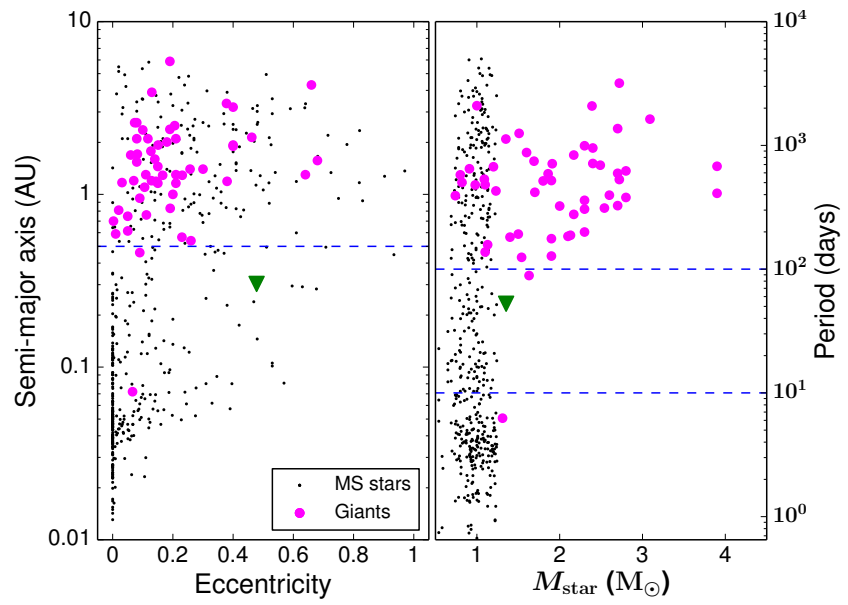

Fig. 3. Left panel: eccentricity and semi-major axis of the extrasolar planets discovered around MS stars (black dots) and giant stars (magenta circles). The dashed line shows the value of $a=0.5 \mathrm{AU}$. Right panel: orbital period versus stellar mass. The dashed lines represent the region of $10 \leq P_{\text {orb }} \leq 100$ days. The position of Kepler-432 b is marked with a green triangle in both panels.

most of the planets around giant stars are found - only $39 \%$ of planets orbiting solar-type stars exhibit $e<0.2$, and a K-S test gives a probability of $0.1 \%$ that the eccentricity of planets around giant and MS stars is drawn from the same distribution.

Because Kepler-432 b is dynamically young (circularization time scale $\tau_{\text {circ }} \sim 150 \mathrm{Gyr}$; see Jackson et al. 2008), the nonzero eccentricity of the planet might be a tracer of its migration history. In this context, it is expected that some kind of high-eccentricity migration (HEM) mechanism might have operated to excite the eccentricity of Kepler-432 b to its current value (see Socrates et al. 2012, and references therein). Within this scenario, it has been proposed that warm Jupiters can form via tidal dissipation at the high-e stage during Kozai-Lidov oscillations (Wu \& Lithwick 2011; Dong et al. 2014). Moreover, 
Dong et al. (2014) suggested that these planets need close companions for HEM to occur. They calculated upper limits for the perturber separation $b_{\text {per }}$ to allow an efficient tidal dissipation of the orbit. Assuming a perturber mass of between 1 and $10 M_{\text {Jup }}$, we find an upper limit of $b_{\text {per }}=6-13 \mathrm{AU}$ for an additional object in the Kepler-432 system.

Following Montet et al. (2014), for example, the detected $\mathrm{RV}$ acceleration $\dot{\gamma}=0.44 \mathrm{~m} / \mathrm{s} / \mathrm{d}$ requires a perturbing object in a circular orbit at $a \sim 1$ and $a \sim 3$ AU for masses of 1 and $10 M_{\text {Jup }}$, respectively, consistent with the HEM scenario. We consider it unlikely that spot-induced variability might be the source of the RV drift because no emission in the core of the $\mathrm{Ca} \mathrm{H}$ and $\mathrm{K}$ lines is detected in the FIES co-added spectrum. Furthermore, McQuillan et al. (2013) found no significant modulation in the Kepler light curve of Kepler-432, which suggests that this is a magnetically quiet star. A second companion in the system might thus account for the observed RV drift. Additional observations are needed to better assess the nature of this trend.

\subsection{Post-main-sequence evolution}

After Kepler-91 b and HIP 67851 b, Kepler-432 b is the third planet found to orbit a giant star at a distance closer than $a=$ $0.5 \mathrm{AU}$ (Fig. 3), and the second found to transit a star ascending the RGB. Although the current sample is not statistically significant, the discovery of Kepler-432 b confirms that closein planets around intermediate-mass giant stars do exist. Given the short dissipation time-scale of protoplanetary disks, gravitational interaction seems to be the favorite migration channel for close-in planets of intermediate-mass stars. Their paucity might be ascribed to enhanced tidal dissipation and subsequent stellar engulfment during the RGB phase.

Villaver \& Livio (2009) and Kunitomo et al. (2011) have computed the planetary orbit evolution during the post MS phase for stars more massive than the Sun. They concluded that shortperiod planets are swallowed by their host stars as a result of the increased star-planet tidal interaction during the RGB phase. Both studies predict a critical semi-major axis below which planets are engulfed by their host star. The expected values for a star as massive as Kepler-432 are much higher than the current semimajor axis of Kepler-432 b. This implies that the planet will not survive the RGB phase and will be swallowed by its host star before it reaches the tip of the RGB.

\section{Conclusions}

We spectroscopically confirmed the planetary nature of the transiting candidate Kepler-432 b, derived a planetary mass of $M_{\mathrm{p}}=5.84 \pm 0.05 M_{\mathrm{Jup}}$, and found that the orbit is eccentric $(e=0.478 \pm 0.004)$. Kepler-432 b is the first bona fide warm Jupiter planet found to orbit a giant star, and, after Kepler-91 b, is the second found to transit a giant star. The semi-major axis $a=0.303 \pm 0.007 \mathrm{AU}$ and eccentricity of the planet suggest that some kind of migration mechanism must have operated (or is operating now) to bring the planet to its current position. In this context, we discussed the high-eccentricity migration scenario as a plausible mechanism for the formation of this system. This possibility, although not directly verifiable with the currently available data, would account for the high eccentricity and small semi-major axis of the planet, provided that a second massive planet is also orbiting the system. The latter scenario is corroborated by the detection of a significant radial velocity trend in our data. Additional spectroscopic follow-up observations are needed to better characterize the system.

Although our discovery confirms that close-in $(a \lesssim 0.5 \mathrm{AU})$ giant planets can exist around giant stars, more detections are needed to properly characterize the population of these objects around post-MS stars. According to current post-MS evolutionary models, Kepler-432 b will not survive the RGB phase and will be engulfed by its host star.

Note added in proof. This Letter was submitted in parallel with that of Ciceri et al. (2015), who independently also confirmed the planetary nature of Kepler-432 b. Their results agree with ours within the observational errors.

Acknowledgements. M.O. and S.R. acknowledge funding from the Deutsche Forschungsgemeinschaft, DFG under project number RE 2694/3-1 611328 to carry out the observations at Calar Alto. H.J.D. and D.N. acknowledge support by grant AYA2012-39346-C02-02 of the Spanish Ministerio de Economía y Competitividad. We are very grateful to the staff members at Calar Alto and at the Nordic Optical Telescope for their valuable and unique support during the observations. M.O. thanks N. Kudryavtseva and S. Ciceri for useful discussion.

\section{References}

Aceituno, J., Sánchez, S. F., Grupp, F., et al. 2013, A\&A, 552, A31 Adamów, M., Niedzielski, A., Villaver, E., et al. 2012, ApJ, 754, 15 Bressan, A., Marigo, P., Girardi, L., et al. 2012, MNRAS, 427, 127 Buchhave, L. A., Bakos, G. A., Hartman, J. D., et al. 2010, ApJ, 720, 1118 Castelli, F., \& Kurucz, R. L. 2004 [arXiv: astro-ph/0405087]

Ciceri, S., Lillo-Box, J., Southworth, J., et al. 2015, A\&A, 573, L5 Currie, T. 2009, ApJ, 694, 171

Döllinger, M. P., Hatzes, A. P., Pasquini, L., et al. 2009, A\&A, 505, 1311

Dong, S., Katz, B., \& Socrates, A. 2014, ApJ, 781, L5

Fortney, J. J., Marley, M. S., \& Barnes, J. W. 2007, ApJ, 659, 1661

Gandolfi, D., Alcalá, J. M., Leccia, S., et al. 2008, ApJ, 687, 1303

Gettel, S., Wolszczan, A., Niedzielski, A., et al. 2012, ApJ, 756, 53

Gray, R. O., \& Corbally, C. J. 1994, AJ, 107, 742

Hekker, S., \& Meléndez, J. 2007, A\&A, 475, 1003

Hekker, S., Snellen, I. A. G., Aerts, C., et al. 2008, A\&A, 480, 215

Huber, D., Chaplin, W. J., Christensen-Dalsgaard, J., et al. 2013, ApJ, 767,

127

Jackson, B., Barnes, R., \& Greenberg, R. 2008, MNRAS, 391, 237

Johnson, J. A., Bowler, B., Howard, A., et al. 2010, ApJ, 721, 153

Johnson, J. A., Payne, M., Howard, A., et al. 2011, AJ, 141, 16

Jones, M., Jenkins, J., Rojo, P., et al. 2013, A\&A, 556, A78

Jones, M. I., Jenkins, J. S., Bluhm, P., et al. 2014, A\&A, 566, A113

Jones, M. I., Jenkins, J. S., Rojo, P., et al. 2015, A\&A, 573, A3

Kane, M. R., \& Gelino, D. M. 2010, ApJ, 724, 818

Kretke, K., Lin, D., Garaud, P., et al. 2009, ApJ, 690, 407

Kunitomo, M., Ikoma, M., Sato, B., Katsuta, Y., \& Ida, S. 2011, ApJ, 737, 66

Lillo-Box, J., Barrado, D., Moya, A., et al 2014, A\&A, 562, A109

López-Morales, M., \& Seager, S. 2007, ApJ, 667, L191

Loyd, R. O. P., \& France, K. 2014, ApJS, 211, 9

McQuillan, A., Mazeh, T., \& Aigrain, S. 2013, ApJ, 775, 11

Montet, B. T., Crepp, J. R., Johnson, J. A., et al. 2014, ApJ, 781, 28

Nowak, G. 2013, Ph.D. Thesis, Nicolaus Copernicus Univ., Toruń, Poland

Queloz, D., Henry, G. W., Sivan, J. P., et al. 2001, A\&A, 379, 279

Reffert, S., Bergmann, C., Quirrenbach, A., et al. 2015, A\&A, submitted

Sato, B., Omiya, M., Harakawa, H., et al. 2013, PASJ, 65, 85

Saumon, D., \& Guillot, T. 2003, ApJ, 609, 1170

Schlaufman, K., \& Winn, J. 2013, ApJ, 772, 143

Seager, S., Kuchner, M., Hier-Majumder, C. A., et al. 2007, ApJ, 669, 1279

Socrates, A., Katz, B., Dong, S., et al. 2012, ApJ, 750, 106

Sudarsky, D., Burrows, A., \& Pinto, P. 2000, ApJ, 538, 885

Sudarsky, D., Burrows, A., Hubeny, I., et al. 2005, ApJ, 627, 520

Telting, J. H., Avila, G., Buchhave, L., et al. 2014, Astron. Nachr., 335, 41

Trifonov, T., Reffert, S., Xianyu, T., et al. 2014, A\&A, 568, A64

Villaver, E., \& Livio, M. 2009, ApJ, 705, 81

Wang, X. S., Wright, J. T., Cochran, W., et al. 2012, ApJ, 761, 46

White, T. R., Bedding, T. R., Stello, D., et al. 2011, ApJ, 743, 161

Wright, J. T., \& Howard, A. W. 2009, ApJS, 182, 205

Wu, Y., \& Lithwick, Y. 2011, ApJ, 735, 109 
M. Ortiz et al.: The transiting warm Jupiter Kepler-432 b

Table 3. CAFE and FIES radial velocity measurements of Kepler-432.

\begin{tabular}{|c|c|c|c|c|c|}
\hline $\begin{array}{c}\text { BJD } \\
(-2450000)\end{array}$ & $\begin{array}{c}R V \\
\mathrm{~km} \mathrm{~s}^{-1}\end{array}$ & $\begin{array}{c}\sigma_{\mathrm{R} V} \\
\mathrm{~km} \mathrm{~s}^{-1}\end{array}$ & $\begin{array}{c}\text { Exp. Time } \\
\text { s }\end{array}$ & $\begin{array}{l}S / N / \text { pixel } \\
\text { @ 5500 }\end{array}$ & $\begin{array}{c}\mathrm{BVS} \\
\mathrm{km} \mathrm{s}^{-1}\end{array}$ \\
\hline \multicolumn{6}{|l|}{ CAFE } \\
\hline 6826.63307 & -36.018 & 0.014 & 5400 & 25 & - \\
\hline 6827.64045 & -36.017 & 0.029 & 5400 & 16 & - \\
\hline 6828.62402 & -35.976 & 0.019 & 5400 & 22 & - \\
\hline 6835.64888 & -35.799 & 0.016 & 5400 & 26 & - \\
\hline 6836.63077 & -35.808 & 0.017 & 5400 & 22 & - \\
\hline 6837.63356 & -35.820 & 0.025 & 5400 & 16 & - \\
\hline 6838.63076 & -35.783 & 0.024 & 5400 & 20 & - \\
\hline 6839.63282 & -35.830 & 0.017 & 5400 & 24 & - \\
\hline 6858.56617 & -36.314 & 0.029 & 5400 & 18 & - \\
\hline 6870.39070 & -36.125 & 0.019 & 5400 & 19 & - \\
\hline 6906.41528 & -36.316 & 0.023 & 4500 & 21 & - \\
\hline \multicolumn{6}{|l|}{ FIES } \\
\hline 6841.71887 & -35.409 & 0.008 & 1800 & 31 & -0.001 \\
\hline 6842.71994 & -35.512 & 0.009 & 1800 & 30 & 0.004 \\
\hline 6843.71619 & -35.610 & 0.007 & 1800 & 33 & -0.002 \\
\hline 6844.72316 & -35.653 & 0.009 & 1680 & 29 & -0.001 \\
\hline 6854.70204 & -35.704 & 0.006 & 2400 & 41 & -0.003 \\
\hline 6855.69674 & -35.693 & 0.008 & 2700 & 44 & -0.007 \\
\hline 6856.68809 & -35.680 & 0.005 & 2700 & 41 & -0.002 \\
\hline 6860.40078 & -35.632 & 0.008 & 1800 & 34 & -0.005 \\
\hline 6875.41274 & -35.418 & 0.007 & 2400 & 36 & 0.006 \\
\hline 6880.52282 & -35.327 & 0.006 & 2400 & 44 & 0.002 \\
\hline 6892.55722 & -35.223 & 0.007 & 2400 & 35 & -0.004 \\
\hline 6901.41459 & -35.716 & 0.007 & 2400 & 36 & -0.006 \\
\hline 6902.46355 & -35.719 & 0.007 & 2400 & 31 & -0.004 \\
\hline 6903.52509 & -35.704 & 0.006 & 2400 & 38 & -0.001 \\
\hline 6916.36055 & -35.565 & 0.013 & 1800 & 24 & 0.012 \\
\hline 6936.46518 & -35.234 & 0.006 & 2400 & 44 & -0.001 \\
\hline
\end{tabular}

Notes. The total exposure time, S/N per pixel at $5500 \AA$, and CCF bisector spans are listed in the last three columns. 